\title{
Towards Terawatt-Scale Spectrally Tunable Terahertz Pulses via Relativistic Laser-Foil Interactions
}

\author{
Guo-Qian Liao $\oplus^{1,2}$ Hao Liu, ${ }^{1,3}$ Graeme G. Scott, ${ }^{2}$ Yi-Hang Zhang $\odot,{ }^{1,7}$ Bao-Jun Zhu, ${ }^{1,7}$ Zhe Zhang, ${ }^{1}$ \\ Yu-Tong Li, ${ }^{1,7,10,11,{ }^{*}}$ Chris Armstrong, ${ }^{4,2}$ Egle Zemaityte, ${ }^{4,2}$ Philip Bradford $\odot{ }^{5}$ Dean R. Rusby, ${ }^{2}$ \\ David Neely, ${ }^{2,4, \dagger}$ Peter G. Huggard $\odot{ }^{6}$ Paul McKenna $\odot,{ }^{4}$ Ceri M. Brenner, ${ }^{2}$ Nigel C. Woolsey $\odot,{ }^{5}$ \\ Wei-Min Wang $\odot{ }^{12,1,8}$ Zheng-Ming Sheng, ${ }^{3,4,8,9}$ and Jie Zhang ${ }^{1,3,8, \sharp}$ \\ ${ }^{1}$ Beijing National Laboratory for Condensed Matter Physics, Institute of Physics, \\ Chinese Academy of Sciences, Beijing 100190, China \\ ${ }^{2}$ Central Laser Facility, STFC Rutherford Appleton Laboratory, Didcot OX11 OQX, United Kingdom \\ ${ }^{3}$ Key Laboratory for Laser Plasmas (Ministry of Education), School of Physics and Astronomy, \\ Shanghai Jiao Tong University, Shanghai 200240, China \\ ${ }^{4}$ Department of Physics SUPA, University of Strathclyde, Glasgow G4 ONG, United Kingdom \\ ${ }^{5}$ Department of Physics, York Plasma Institute, University of York, York YO10 5DD, United Kingdom \\ ${ }^{6}$ RAL Space, STFC Rutherford Appleton Laboratory, Didcot OX11 OQX, United Kingdom \\ ${ }^{7}$ School of Physical Sciences, University of Chinese Academy of Sciences, Beijing 100049, China \\ ${ }^{8}$ Collaborative Innovation Center of IFSA (CICIFSA), Shanghai Jiao Tong University, \\ Shanghai 200240, China \\ ${ }^{9}$ Tsung-Dao Lee Institute, Shanghai 200240, China \\ ${ }^{10}$ Songshan Lake Materials Laboratory, Dongguan, Guangdong 523808, China \\ ${ }^{11}$ CAS Center for Excellence in Ultra-intense Laser Science, Shanghai 201800, China \\ ${ }^{12}$ Department of Physics, Renmin University of China, Beijing 100872, China
}

(Received 17 July 2019; revised 4 June 2020; accepted 24 July 2020; published 18 September 2020)

An ever-increasing number of strong-field applications, such as ultrafast coherent control over matter and light, require driver light pulses that are both high power and spectrally tunable. The realization of such a source in the terahertz $(\mathrm{THz})$ band has long been a formidable challenge. Here, we demonstrate, via experiment and theory, efficient production of terawatt (TW)-level $\mathrm{THz}$ pulses from high-intensity picosecond laser irradiation on a metal foil. It is shown that the $\mathrm{THz}$ spectrum can be manipulated effectively by tuning the laser pulse duration or target size. A general analytical framework for $\mathrm{THz}$ generation is developed, involving both the high-current electron emission and a time-varying electron sheath at the target rear, and the spectral tunability is found to stem from the change of the dominant $\mathrm{THz}$ generation mechanism. In addition to being an ultrabright source (brightness temperature of about $10^{21} \mathrm{~K}$ ) for extreme $\mathrm{THz}$ science, the $\mathrm{THz}$ radiation presented here also enables a unique in situ laser-plasma diagnostic. Employing the THz radiation to quantify the escaping electrons and the transient sheath shows good agreement with experimental measurements.

DOI: 10.1103/PhysRevX.10.031062

\section{INTRODUCTION}

Generally, the spectral range and brightness of a light source define its applicability. In this sense, the emerging research field of extreme terahertz $(\mathrm{THz})$ science [1]

\footnotetext{
*ytli@iphy.ac.cn

†david.neely@stfc.ac.uk

jzhang1@sjtu.edu.cn
}

Published by the American Physical Society under the terms of the Creative Commons Attribution 4.0 International license. Further distribution of this work must maintain attribution to the author(s) and the published article's title, journal citation, and DOI.
Subject Areas: Plasma Physics

primarily benefits from recent advances made in high-power $\mathrm{THz}$ sources [2,3]. Spectrally tunable, high-brightness $\mathrm{THz}$ sources are highly desired for numerous applications, such as selectively driving and controlling specific low-energy degrees of freedom in matter [4,5], waveguide-couplingbased $\mathrm{THz}$ electron accelerators and manipulators [6-10], and nonlinear $\mathrm{THz}$ bioeffects studies [11]. Currently, electron accelerators and ultrafast lasers are the two main drivers for generating intense $\mathrm{THz}$ pulses [12]. Sophisticated manipulation of electron beams or undulators is required in accelerators to modify the $\mathrm{THz}$ spectra [13-17]. For THz sources based on laser optical rectification [18-21] or photoconductive antennas [22], the spectral coverage is mainly determined by the properties of optical crystals 
or photoconductors used, and the energy scalability is limited because of the low optical damage threshold of the emitters [3]. Laser-produced plasma is an alternative promising emitter of high-power THz pulses [23,24]. For the scheme of laser filamentation in gases [25-27] or liquids $[28,29]$, the resulting $\mathrm{THz}$ radiation is ultrabroadband (up to about $50 \mathrm{THz}$ ), and the saturation of $\mathrm{THz}$ energy has been observed in air plasmas [25-27]. Relativistic laser interactions with solid targets are attracting increasing attention as a novel $\mathrm{THz}$ source because of the high $\mathrm{THz}$ yield produced without the tendency to saturate [30]. Very recently, multimillijoule $\mathrm{THz}$ pulses have been demonstrated experimentally [31]. For the THz emissions from the rear side of a solid target [31-40], a number of studies have been reported on the influence of laser and target parameters on the $\mathrm{THz}$ radiation, and several distinct models have been proposed to understand the specific experimental observations. Nevertheless, the distinctions of these models and their respective contributions to the global $\mathrm{THz}$ radiation remain elusive, even under comparable laser conditions [32-40]. It should also be noted that almost all of the relevant experiments are performed in the femtosecond laser regime [32-40], and little attention has been given specifically to the control of $\mathrm{THz}$ spectra. Apparently, it is essential to investigate the $\mathrm{THz}$ properties in a broader parameter space for better understanding THzgeneration physics and broadening $\mathrm{THz}$ applications.

Here, we study, theoretically and experimentally, the $\mathrm{THz}$ generation in a new regime, where a tunable intense picosecond laser pulse interacts with a metallic foil target of varying lateral size. Besides advancing the $\mathrm{THz}$ peak power towards an unprecedented terawatt (TW) level, we also demonstrate that the $\mathrm{THz}$ spectrum can be tuned by varying the laser pulse duration or target size. With a comprehensive theoretical framework proposed, the spectral tunability is attributed to the competition of various $\mathrm{THz}$-generation processes, and the $\mathrm{THz}$ radiation is applied to diagnose the electron emission and the transient sheath.

\section{THEORY AND MODELING}

Energetic electron emission and ion acceleration have been suggested, separately [31-35] or jointly [36-40], to be responsible for the $\mathrm{THz}$ generation at the rear side of laserirradiated solid targets. Nevertheless, how to identify the respective roles played by electrons and ions under diverse conditions remains an open question [37]. To investigate the tunability of THz spectral properties, it is imperative to improve our understanding of the THz-generation physics and develop a comprehensive self-consistent analytical framework for various $\mathrm{THz}$-generation scenarios.

From a physical point of view, time-varying currents produce electromagnetic waves. For a metal target, the long-wavelength $\mathrm{THz}$ radiation can only originate from the target-vacuum interface. During ultraintense laser interactions with solids, a mega-ampere current of energetic electrons is generated at the front side of the target [41], and it propagates to the rear side if the target is not sufficiently thick. Three kinds of transient currents can be excited successively as a consequence of the passage of energetic electrons across the target's rear surface. Owing to the dielectric discontinuity at the target-vacuum boundary, on one hand, a transverse current consisting of background cold electrons is induced, emitting electromagnetic radiation referred to as the transition radiation (TR) $[34,42,43]$. On the other hand, a strong electrostatic field is built up by the transient charge imbalance at the target surface. This sheath field ionizes surface atoms and accelerates ions [44] while reflecting low-energy electrons back into the target. For those electrons trapped in the sheath, whether the deceleration and acceleration in the sheath fields [35] or the leaving and reentering the target will induce transient emission, hereafter termed bremsstrahlung-like radiation (BR). This scenario was not considered previously in conventional accelerators [16] and gas plasmas [24] because of the absence of strong sheath fields. After laser interactions, the net charge in the sheath decays over time, and as a result, the moving electron sheath behaves like a longitudinal transient current, thereby giving rise to electromagnetic radiation referred to as the sheath radiation (SR) [36-40]. Figure 1(a) sketches these radiation scenarios.

We have derived analytical descriptions of these radiation processes (see Sec. VII for details on analytical models). Our modeling shows that the radiation generated from different processes has distinctly different spectral profiles [see Fig. 1(b)]. The TR produced by the escaping electron bunch is concentrated at the low-frequency end of the spectrum. A longer electron bunch duration will result in a narrower spectral width. In contrast, the SR spectrum shows an asymmetrically peaked distribution, with a central frequency inversely proportional to the decay time of the sheath. The spectrum of the BR produced by the trapped electrons is related not only to the bunch form factor (determined by the electron bunch duration) but also to the dwell time of electrons in the sheath (dictated jointly by the sheath field strength and electron energy) [see Eq. (2) in Sec. VII]. Usually, the femtosecond or picosecond laseraccelerated energetic electron bunch has an approximately equal duration to the laser pulse [45], and the sheath driven by a burst of electrons evolves on a picosecond timescale [46]. Hence, the resulting radiation, whether it is TR, BR, or $\mathrm{SR}$, could fall in the $\mathrm{THz}$ regime. As the final $\mathrm{THz}$ radiation is composed of these components, it is possible to tune the THz spectrum by controlling the TR, BR, or SR separately, or by varying their relative contributions.

To elaborate on the potential for $\mathrm{THz}$ spectral tunability, we numerically calculate the TR, BR, SR, and the synthetic $\mathrm{THz}$ radiation spectra as a function of the electron bunch duration [Figs. 1(c) and 1(d)], by substituting reasonable parameters into the analytical models (see Sec. VII for the 
(a)
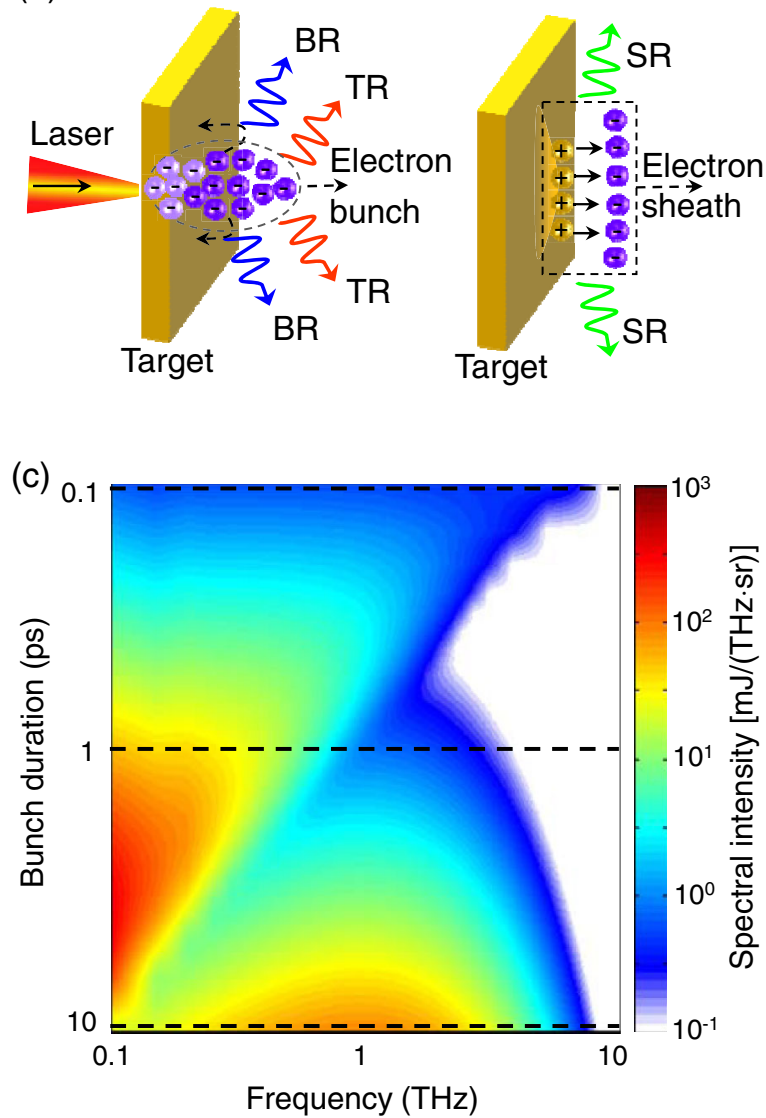

(b)

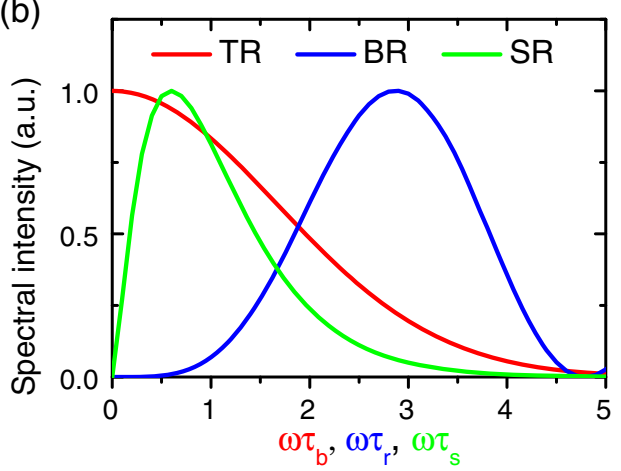

(d)

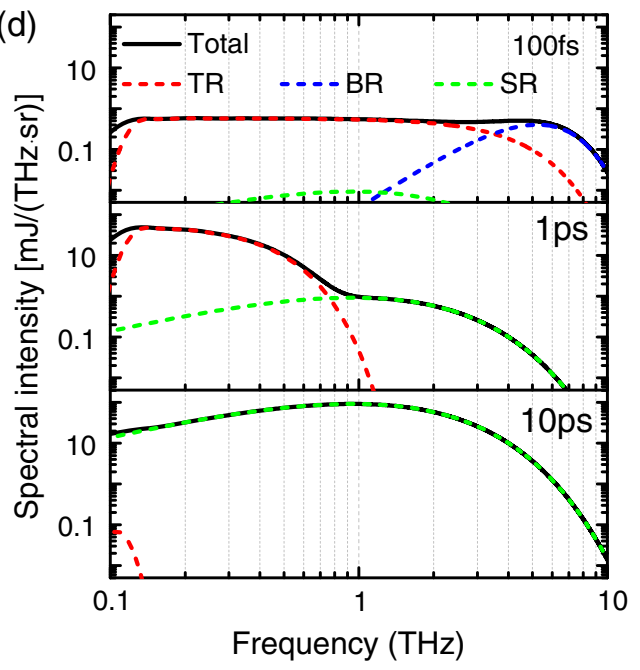

FIG. 1. Model illustration and theoretical projections. (a) Schematic illustration of the THz-generation scenarios at the rear side of a laser-irradiated foil. Left diagram: TR induced by escaping electrons and BR by electrons trapped in the sheath. Right diagram: SR induced by the time-varying electron sheath. (b) Spectral profiles for the TR (red) and the BR induced by a single electron (blue), and the SR (green). All curves are normalized to unity at their peaks. Here, $\tau_{b}, \tau_{r}$, and $\tau_{s}$ in the horizontal axis refer to the electron bunch duration, half the dwell time of a single electron in the sheath, and the characteristic decay time of the sheath, respectively. (c) Resultant radiation spectra from TR, BR, and SR, calculated at given parameters (see Sec. VII), as a function of the electron bunch duration. (d) Three lineouts (at $100 \mathrm{fs}, 1 \mathrm{ps,}$ and $10 \mathrm{ps)} \mathrm{taken} \mathrm{from} \mathrm{the} \mathrm{spectral} \mathrm{intensity} \mathrm{map} \mathrm{shown} \mathrm{in} \mathrm{panel} \mathrm{(c).} \mathrm{Dashed} \mathrm{curves} \mathrm{represent} \mathrm{the}$ individual spectral components from different radiation processes. The BR is too weak to appear on the 1-ps and 10-ps plots.

parameter details). The calculation is performed on the premise that the electron bunch current remains constant (corresponding to a constant laser intensity and laser absorption efficiency in the experiment). In the regime of femtosecond bunch duration, TR dominates the THz generation. As the electron bunch duration increases to several picoseconds, the TR induced by the escaping electrons grows in spectral intensity and narrows in spectral width. When the bunch duration is greater than around $10 \mathrm{ps}$, the majority of the TR energy goes into the spectral region below $0.1 \mathrm{THz}$, reducing the spectral intensity in the generally accepted $\mathrm{THz}$ range $(0.1-10 \mathrm{THz})$. The BR intensity decreases significantly with increasing bunch duration because the redshifted bunch form factor [red curve in Fig. 1(b)] will overlap less and less with the relatively high-frequency radiation of a single trapped electron [blue curve in Fig. 1(b)]. In contrast, the SR intensity increases with the square of the bunch duration. In the regime of picosecond bunch duration, the SR separates spectrally from the redshifted TR and gradually exceeds the TR in intensity. Eventually, for a multipicosecond bunch duration, SR becomes the dominant THz-generation process. Our modeling predicts that by tuning the bunch duration, one can change the contributions of different radiation processes and thus reshape the global $\mathrm{THz}$ spectrum.

\section{EXPERIMENTAL DEMONSTRATION}

Most of the previous experiments on $\mathrm{THz}$ generation from the rear side of solid targets were performed with tensof-femtoseconds laser pulses [32-40]. According to our modeling, the TR dominates the $\mathrm{THz}$ generation in this case, in line with previous experimental and theoretical investigations $[34,36]$. In principle, the TR spectrum can be narrowed by increasing the pulse duration [31,43]. To validate the proposed models and the predicted spectral 

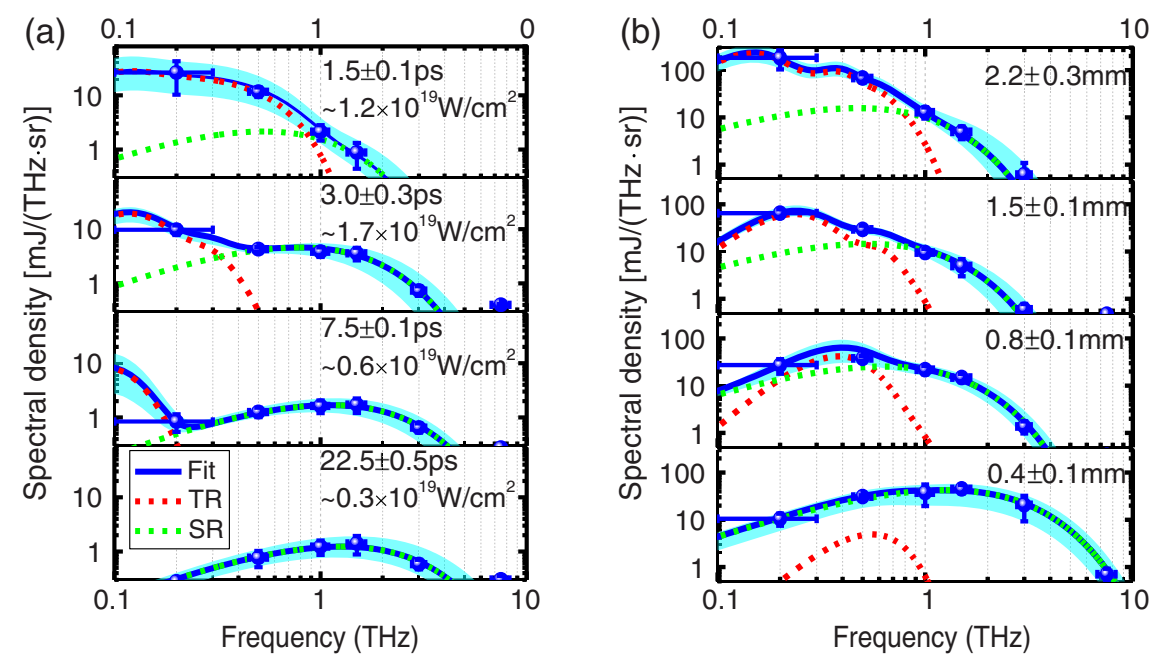

FIG. 2. Experimental results and theoretical fitting. (a) THz spectra for given laser pulse durations and intensities at a constant target size of about $3 \mathrm{~mm}$. (b) THz spectra for different target sizes under similar laser conditions (laser energy of about $70 \mathrm{~J}$ and pulse duration of about $1.5 \mathrm{ps}$ ). Circles are the experimental data. Solid curves are fits using the analytical models described in the preceding section. Dashed curves correspond to the decomposition of the resultant THz spectra into the TR (red) and SR (green) components, and the cyan band shows the fitting range to cover the errors in the experimental data (see Sec. VII for details on the spectral measurements and fitting). Vertical error bars are a combination of the shot-to-shot fluctuations in the drive laser parameters and the measurement uncertainty in the detectors. Horizontal error bars represent the transmission bandwidth of the filters used.

tunability, we extend the laser parameter space explored experimentally to the picosecond regime over which the $\mathrm{THz}$ spectrum is parametrically studied (see Sec. VII for details on the experimental configuration and data acquisition). In the absence of a femtosecond probe, we measure the discretized $\mathrm{THz}$ spectrum by using a set of bandpass filters.

Two sets of parameter scans are carried out in the experiment. To directly compare with the theoretical predictions shown in Fig. 1(c), we first incrementally change the laser pulse duration from $1.5 \mathrm{ps}$ to $22.5 \mathrm{ps}$. The measured $\mathrm{THz}$ spectra vary markedly with the laser pulse duration [see Fig. 2(a)]. The variation tendency is in good accordance with the theoretical prediction, despite the fluctuation in laser intensity. At $1.5 \mathrm{ps}$, a monotonically decreasing spectral profile is observed. As expected, the spectral coverage of less than $3 \mathrm{THz}$ is much narrower than that (about $30 \mathrm{THz}$ ) measured in the femtosecond laserdriven case [33,34]. With increasing laser pulse duration, the $\mathrm{THz}$ component below $0.5 \mathrm{THz}$ decreases significantly and even becomes unobservable at $20 \mathrm{ps}$, while the components greater than $1 \mathrm{THz}$ are enhanced gradually, though at a reduced laser intensity, leading to an emerging spectral peak at $0.5-2 \mathrm{THz}$.

The TR model predicts that the finite transverse size of the boundary can suppress the low-frequency radiation [43]. A previous investigation also shows that the limited target surface area can affect the sheath dynamics [47]. In this regard, another corollary to our modeling is that by tuning the target size, we would also be able to tailor the $\mathrm{THz}$ spectra. To verify this case, we have varied the lateral target size while keeping the laser parameters (energy and pulse duration) approximately fixed. The measurements show that the THz spectrum is strongly dependent on the target size [Fig. 2(b)]. With decreasing target size, the THz component within $0.5 \mathrm{THz}$ decreases substantially, while, instead, the spectral intensity above $1 \mathrm{THz}$ increases. At a target size of about $0.8 \mathrm{~mm}$ and smaller, the $\mathrm{THz}$ spectrum turns into a nonmonotonic profile with a central frequency in the range of $0.5-2 \mathrm{THz}$.

The experimental data can be well reproduced by the analytical models (see Sec. VII for details on the fitting of the experimental $\mathrm{THz}$ spectra), based on which one can identify the respective contribution of TR and SR (see the curves in Fig. 2). As the laser pulse duration increases, the TR shifts towards the low-frequency end, separating from the SR-induced spectrum. Meanwhile, the SR is also tuned, both in intensity and spectrum, as a result of the variation in the electron sheath (the dependence of sheath electrons on the pulse duration and target size will be discussed later). In the scan of the target size, the TR reduces in intensity with a reduction in target size, while the SR is enhanced and blueshifted in spectrum for the $0.8-\mathrm{mm}$ and $0.4-\mathrm{mm}$ targets (the reason will be explained in Sec. V). The good fit to the experimental results not only validates the analytical models but also suggests that it is feasible to tune the $\mathrm{THz}$ spectrum in a predictable manner.

\section{FIGURES OF MERIT}

In light of the extrapolation of experimental measurements with theoretical models, one can evaluate some 
TABLE I. Comparison with state-of-the-art high-power THz sources, including a $\mathrm{THz}$ free-electron laser (FEL), TR in a linear accelerator (LINAC), optical rectification (OR) in organic crystals and lithium niobate $\left(\mathrm{LiNbO}_{3}\right)$, and laser-induced plasmas in air, liquids, and solids. All sources are broadband except the FEL. The data for existing sources are referenced from the previously reported experimental records.

\begin{tabular}{|c|c|c|c|c|c|}
\hline & & $\begin{array}{c}\text { Peak } \\
\text { power }(\mathrm{GW})\end{array}$ & $\begin{array}{c}20-\mathrm{dB} \\
\text { bandwidth }(\mathrm{THz})\end{array}$ & $\begin{array}{c}\text { Brightness } \\
\text { temperature }\left(10^{19} \mathrm{~K}\right)\end{array}$ & $\begin{array}{c}\text { Laser-THz conversion } \\
\text { efficiency }\end{array}$ \\
\hline FEL [15] & & 0.001 & 0.007 & 1 & $\ldots$ \\
\hline LINAC (TR) [16] & & $2-4$ & $3-10$ & $1-6$ & $\cdots$ \\
\hline Organic crystal (OR) $[18,19]$ & & 4 & 3 & 10 & $3 \%$ \\
\hline $\mathrm{LiNbO}_{3}(\mathrm{OR})[20]$ & & 0.2 & 0.4 & 2 & $0.77 \%(3.8 \%[21])$ \\
\hline Air plasma $[25,26]$ & & 0.1 & 10 & 0.02 & $0.01 \%(0.1 \%[27])$ \\
\hline Liquid plasma [28] & & 0.04 & 15 & 0.005 & $0.1 \%$ \\
\hline \multirow[t]{2}{*}{ Solid plasma } & JETI [33] & 1 & 1.5 & 0.4 & $0.08 \%$ \\
\hline & This work & $(0.08-1.2) \times 10^{3}$ & $(0.5-5)$ & $(0.9-1.9) \times 100$ & $(0.16-0.29) \%$ \\
\hline
\end{tabular}

typical figures of merit for the $\mathrm{THz}$ source demonstrated in the experiment (see Sec. VII for details on the evaluation procedure) and compare with other representative, currently available, high-power sources (see Table I). The detailed data on the evaluation of $\mathrm{THz}$ parameters are summarized in the Supplemental Material [48]. For the irradiation of a copper foil of limited size with a 1.5-ps laser pulse of about $70 \mathrm{~J}$, the peak power of the total $\mathrm{THz}$ radiation emitted from the target rear surface can reach an unprecedentedly high TW level, and the spectrum can be tuned widely in the bandwidth and central frequency across a few $\mathrm{THz}$ by varying the foil size. In contrast, since the $\mathrm{THz}$ spectrum from crystal-based sources depends primarily on the crystal, and the $\mathrm{THz}$ radiation from laser-induced plasmas in air and liquids is ultrabroadband, the opportunity to spectrally tune these sources is small. To characterize the average spectral intensity over the bandwidth, the parameter brightness temperature [49] $T_{B}$ is adopted and calculated as $k_{B} T_{B}=$ Peak power $/\left[\left(\mathrm{M}^{2}\right)^{2} \cdot\right.$ Bandwidth $]$, where $\mathrm{M}^{2}$ is the beam quality factor. The $T_{B}$ evaluated for the presented source is nearly 2 orders of magnitude higher than previous sources. Additionally, the laser-to- $\mathrm{THz}$ energy conversion efficiency reaches up to a subpercentage level, which is comparable to that of crystal-based sources [20]. Despite the broad THz angular distribution (see Fig. S1 in Supplemental Material [48]), we find that a significant fraction, up to $80 \%$, of the THz energy would be collected if using an ellipsoidal mirror with a large-acceptance solid angle [37].

Compared to the very recently reported experiment [31], the peak power evaluated here is nearly doubled. This result is mainly due to, besides the higher pump laser intensity and the optimized $\mathrm{THz}$ detection system, the enhancement of the higher-frequency (thus shorter-pulse-width) SR components at smaller target sizes used here.

\section{APPLICATION AS A LASER-PLASMA DIAGNOSTIC}

In conventional accelerators and laser-wakefield acceleration from gas targets, $\mathrm{THz}$ transition radiation has been widely exploited to diagnose the electron bunch length [50-52], and very recently, it has been used to estimate the bunch size in laser-solid interactions [40] because the $\mathrm{THz}$ radiation inherently carries some characteristics of the driving electrons. From this perspective, besides being a powerful $\mathrm{THz}$ source, the $\mathrm{THz}$ radiation observed in our experiment also offers a versatile diagnostic of both the electron emission and the transient sheath at the target rear. Here, from the fitting of the experimental $\mathrm{THz}$ spectra with our proposed models (curves in Fig. 2), we retrieve laserplasma characteristic quantities, such as the escaping electron bunch charge, and the electron density and charge in the sheath associated with ion acceleration (see Fig. 3).

Since the electrons trapped in the sheath contribute little to the $\mathrm{THz}$ radiation in the current picosecond bunch case, the TR component mainly reflects the properties of the electron bunch escaping the target. A linear relationship is observed between the retrieved and measured escaping electron charges [Fig. 3(a)]. The measured values are higher than the inferred ones because the TR in the THz regime only diagnoses the fast electron bunch emitted in a picosecond-scale duration during the laser-foil interaction. In contrast, the time-integrated experimental measurement also includes the slow electrons emitted during the subsequent plasma expansion and target discharge [53,54], which lasts on a nanosecond timescale and corresponds to the generation of microwaves [55], i.e., frequencies in the $\mathrm{GHz}$ domain.

Given the fact that the SR spectral shape is determined by the sheath decay time, which is, in turn, dependent on the electron density inside the sheath [56], one can retrieve the sheath electron density from the measured SR component [see Fig. 3(b)]. For the case in which a laser pulse of about $10^{19} \mathrm{~W} / \mathrm{cm}^{2}$ and 1.5 -ps interacts with a foil target greater than $1 \mathrm{~mm}$, the sheath electron density is inferred to be of the order of $10^{20} \mathrm{~cm}^{-3}$, which is consistent with previous measurements using optical probing $[57,58]$ at a similar laser intensity. Interestingly, the sheath electron density increases significantly for a target size smaller than 

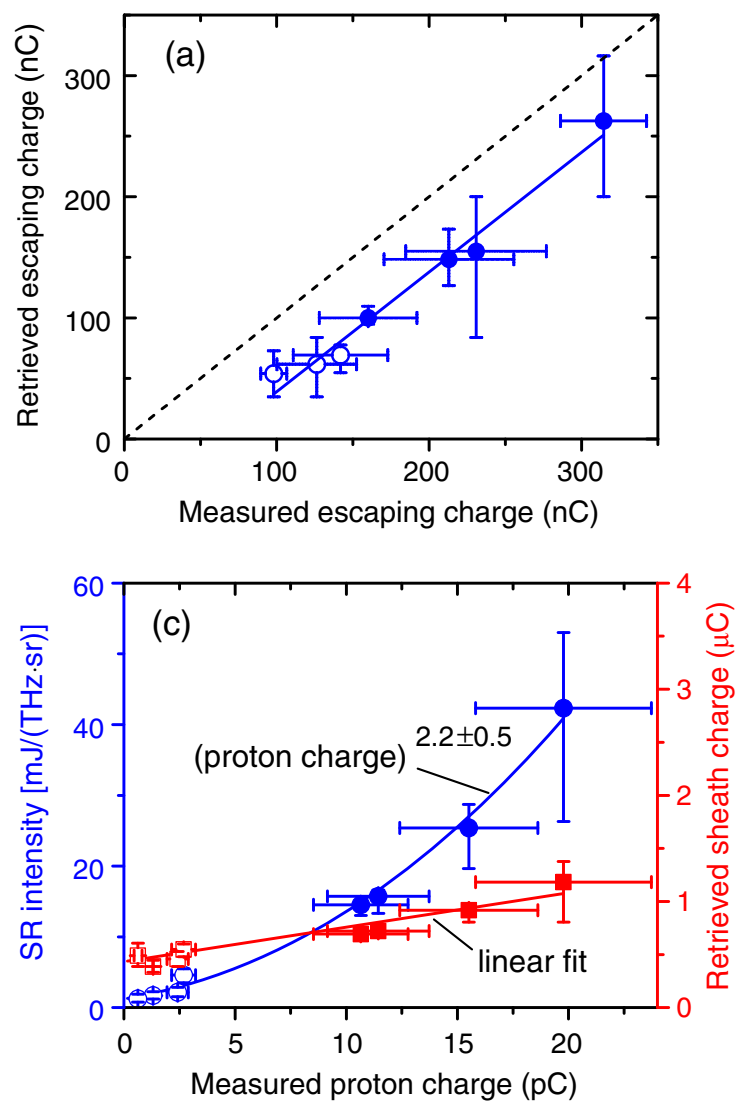
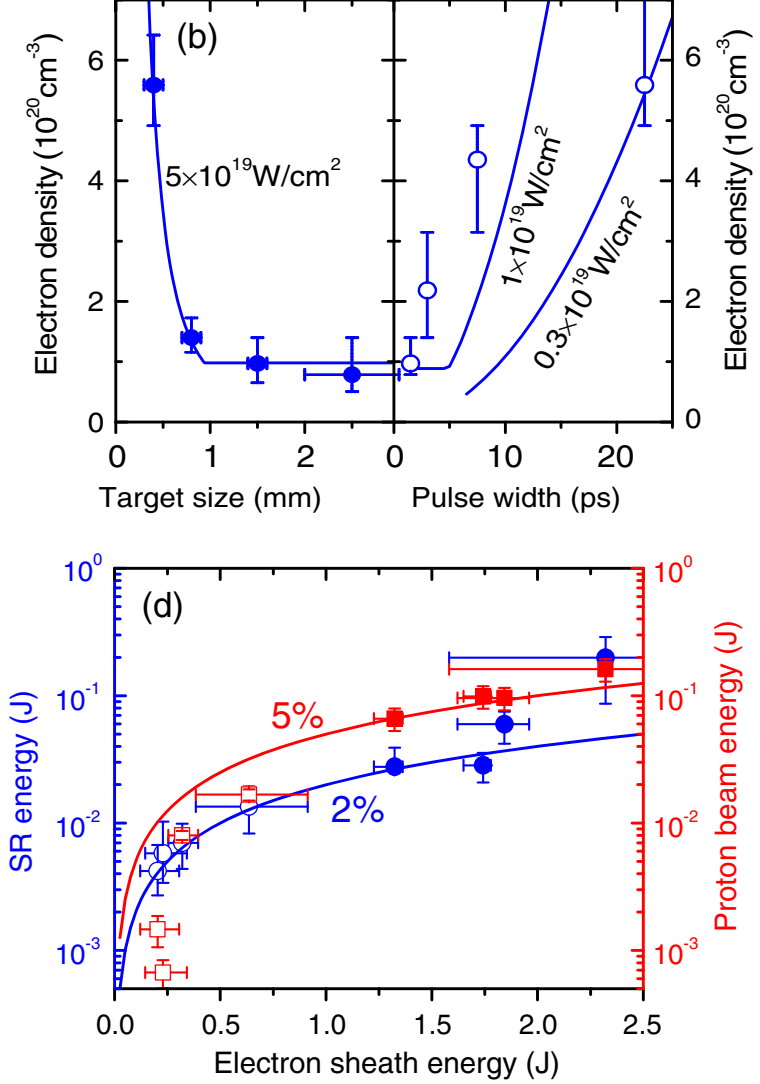

FIG. 3. Retrieval of laser-plasma characteristic quantities from the measured THz spectra. (a) Retrieved bunch charge of the escaping electrons as a function of the experimentally measured values. The solid line shows the linear fit to the data, and the dashed diagonal represents the retrieved values being equal to the measured ones. (b) Retrieved sheath electron density as a function of the target size and laser pulse duration. The curves represent theoretical evaluations at the constant laser energy absorption efficiency of $30 \%$ and indicated laser intensities. (c) Retrieved SR peak spectral intensity (blue circle) and sheath charge (red square), and their dependence on the proton charge measured with an ion spectrometer. The curves represent the power-law fit (blue) and linear fit (red), respectively. (d) Total SR energy (blue circle) and proton beam energy (red square) as a function of the retrieved electron sheath energy. The curves show the given energy conversion efficiency from the sheath. Solid and open marks represent the data set obtained, respectively, in the scan of target size [Fig. 2(b)] and pulse duration [Fig. 2(a)]. See Sec. VII for details on the experimental measurements of electrons and protons and the data analysis. Error bars on the retrieved values are the fitting uncertainties that correspond to the cyan band shown in Fig. 2 . Error bars on the experimental values represent the shot-to-shot fluctuations.

$1 \mathrm{~mm}$ or when increasing the laser pulse duration. This increase can be explained by the transverse electron refluxing effect $[47,59]$, where electrons transported along the target surface are reflected from the target edges during the laser pulse, resulting in a denser electron sheath [60]. The theoretical calculation takes this effect into account (see Sec. VII) and reproduces the trend of the inferred ones. The good agreement between theory and experiment implies that the laser-to-electron energy conversion efficiency is about $30 \%$. The relatively large deviation in the long laser pulse cases could be due to the lack of consideration of the longitudinal electron recirculation effect [61], where the long-duration laser pulse can accelerate the recirculated electrons from the target rear multiple times, resulting in a higher laser absorption efficiency and a denser electron sheath.

Using the SR spectral intensity, one can retrieve the sheath charge. Results in Fig. 3(c) show that the SR intensity has an approximately quadratic dependence on the measured proton charge, and the proton charge is roughly proportional to the sheath charge. This case is consistent with the well-known target-normal sheath acceleration model [62]. Suppose that the electrons in the sheath have the same temperature as the escaping electron bunch, one can then deduce the energy of the electron sheath. We find that the laser-to-sheath energy conversion efficiency is about $(3 \pm 1) \%$ in the target size scan of our experiment, in agreement with previously published values [58]. Considering the angular distribution of SR and the measured proton beam profile, one can evaluate the total energy of the SR and proton beam emitted from the target rear surface. It is found that the SR energy is comparable to the proton energy, both of which are a few percent of the sheath energy [Fig. 3(d)]. 
Optical self-emission, or auxiliary proton and optical probes were previously used to diagnose the transient processes at the target rear surface. Optical transition radiation indicates the microbunched high-energy tail of the electron distribution [63], while the THz TR component corresponds to the global envelope of the escaping electron bunch. It is usually complicated to resolve the target-rear sheath experimentally, either by indirectly probing the quasistatic electric or magnetic fields with proton deflectometry [46], or through high-resolution measurements of the plasma density near the target surface with optical reflectometry [57] or interferometry [58]. Compared to those diagnostics, the SR component provides a direct and quantitative barometer of the electrons localized in the sheath. Moreover, the THz radiation is a more compatible and straightforward diagnostic since neither additional probe beams nor delicate spatiotemporal synchronization is required.

\section{CONCLUSIONS AND OUTLOOK}

In summary, we present experimental and theoretical results on the efficient generation of ultrahigh-power tunable $\mathrm{THz}$ radiation by a high-intensity laser pulse irradiating a foil target. Spectrally-resolved measurements shed light on the long-standing problem of THz-generation mechanisms. Our results show that the $\mathrm{THz}$ radiation is produced via a hybrid TR-SR process. By varying the laser pulse duration or target size, one can control the transition between TR- and SR-dominated THz-generation scenarios, and thereby manipulate the $\mathrm{THz}$ spectrum. Analytical models are proposed to understand the hybrid processes. The good agreement of the models with the experimental results indicates that our theory can describe the essential physics of $\mathrm{THz}$ generation, although some simplifications are applied, such as the adoption of the one-dimensional isothermal plasma-expansion model [56] without considering the time-dependent and multidimensional effects $[64,65]$.

In comparison with other schemes for generating intense $\mathrm{THz}$ pulses, the approach demonstrated here stands out in terms of the peak power ( TW) and spectral brightness (brightness temperature greater than $10^{21} \mathrm{~K}$ ), as well as its broadband spectral tunability. Such a THz source may open up new avenues for nonlinear $\mathrm{THz}$ field-matter interactions [66], compact particle accelerators [67], and multidimensional pump-probe experiments [68] that are operated at a low repetition rate. Moreover, the unprecedentedly high peak power could allow access to a fully new paradigm of relativistic optics [69] in the $\mathrm{THz}$ regime, where there should be an abundance of new physics, as evidenced by the recently emerging ultra-intense laser interactions at midinfrared wavelengths [70-72].

Our results also indicate that the $\mathrm{THz}$ radiation can serve as a unique diagnostic capable of simultaneously characterizing the escaping electron bunch and the electron sheath at the target rear. If we resort to nonscanning $\mathrm{THz}$ waveform measurements [73], it is possible to perform a real-time quantitative diagnosis of the dynamics of electron emission and ion acceleration, which will add significantly to the comprehensive understanding of laser-plasma physics.

\section{METHODS}

\section{A. Analytical models}

TR occurs when the laser-accelerated electrons pass through the target surface, lasting for a duration similar to the laser pulse. Based on the different groups of electrons involved, the source of TR can be divided into two parts: one induced by those electrons that can escape the target into the vacuum (referred to as escaping electrons), the other from electrons that are trapped in the sheath (referred to as trapped electrons). Since the spatial scale of the electron sheath ( $\sim \mu \mathrm{m}$ Debye length) is far less than the formation length of the $\mathrm{THz}$ transition radiation $(\sim 100 \mu \mathrm{m}$ at $\mathrm{THz}$ wavelengths), the TR induced by the escaping electrons can be treated with the conventional model, which has been intensively studied theoretically $[42,43]$. For an electron bunch traversing a metal surface, the radiation energy $I_{\mathrm{TR}}$, emitted in unit angular frequency and unit solid angle, is expressed as

$$
I_{\mathrm{TR}}=N_{e}\left[1+\left(N_{e}-1\right)\left|F\left(\omega, \tau_{b}\right) \cdot T(\rho)\right|^{2}\right]\left\langle I_{\mathrm{TR}}^{e}\right\rangle
$$

where $Q_{\mathrm{esc}}=N_{e} e$ is the bunch charge, $F\left(\omega, \tau_{b}\right)$ is the bunch form factor determined by the bunch duration $\tau_{b}$, $T(\rho)$ describes the effect of finite transverse boundary size $\rho, I_{\mathrm{TR}}^{e}$ is the radiation energy of a single electron, and the angle brackets $\langle\ldots\rangle$ denote an ensemble average over the bunch energy distribution. The divergence of the electron bunch will broaden the radiation spectrum [42]. When fitting the experimental spectra, we take the relative spectral broadening $\delta \omega / \omega=1$ according to the measured angular distribution of the electron bunch, and the transverse bunch profile at the target surface is not considered because it is much smaller than the THz wavelength.

For the BR, a simplified scenario is considered. A beam of $N_{\text {trap }}$ electrons perpendicularly leaves the target surface, and a uniform electrostatic sheath field $E_{\mathrm{sh}}$ exists along the rear target-normal direction. In addition, the deceleration and acceleration of electrons in the sheath field produce transient emission, and electrons leaving and reentering the target will also induce TR-like radiation. To include this radiation, the $\mathrm{BR}$ is modeled as the emission induced by the variation of an electric dipole consisting of the electron and its image charge. For an electron with a momentum of $p$, its velocity becomes zero at $t=\tau_{r}=p / e E_{\mathrm{sh}}$, and it returns to the target at $t=2 \tau_{r}$. The equivalent current of $N_{\text {trap }}$ electrons can be written as $\boldsymbol{J}(\boldsymbol{x}, t)=\Sigma-e \boldsymbol{v}_{j}\left(t_{j}\right)\left\{\delta\left[\boldsymbol{x}-\left(\boldsymbol{R}_{0}+\boldsymbol{r}_{j}\left(t_{j}\right)\right)\right]+\right.$ $\left.\delta\left[\boldsymbol{x}-\left(\boldsymbol{R}_{0}-\boldsymbol{r}_{j}\left(t_{j}\right)\right)\right]\right\}, 0 \leq t_{j} \leq 2 \tau_{r j}$, where $\delta(\ldots)$ represents the Dirac delta function, $\boldsymbol{R}_{0}$ is the vector from the electron 
emission point to the observation point, and $\boldsymbol{v}_{j}$ and $\boldsymbol{r}_{j}$ correspond, respectively, to the time-dependent velocity and trajectory of the $j$ th electron. According to classical electrodynamics [74], the spectral intensity of the radiation produced by $\boldsymbol{J}(\boldsymbol{x}, t)$ is derived, after some calculation, as

$$
\begin{aligned}
I_{\mathrm{BR}}= & {\left[N_{\text {trap }}\left\langle\left|G_{\mathrm{BR}}\left(\omega \tau_{r}, u, \theta\right)\right|^{2}\right\rangle\right.} \\
& +N_{\text {trap }}\left(N_{\text {trap }}-1\right) \mid\left\langle\left. F\left(\omega, \tau_{b}\right) \cdot G_{\mathrm{BR}}\left(\omega \tau_{r}, u, \theta\right) e^{\left.i \omega \tau_{r}\right\rangle}\right|^{2}\right] I_{\mathrm{BR}}^{e}
\end{aligned}
$$

where $Q_{\text {trap }}=N_{\text {trap }} e$ is the trapped electron charge, $I_{\mathrm{BR}}^{e}=e^{2} \sin ^{2} \theta / 4 \pi^{3} \varepsilon_{0} c, \quad u=p / m_{e} c, \quad G_{\mathrm{BR}}(\omega, u, \theta)=$ $\omega \int_{-1}^{1} d \xi e^{i \omega \xi} u \xi \cos \left\{\omega\left[\sqrt{1+u^{2}}-\sqrt{1+u^{2} \xi^{2}}\right] \cos \theta / u\right\} /$ $\sqrt{1+u^{2} \xi^{2}}$, and $\theta$ is the observation angle with respect to the rear target normal.

The SR occurs mainly after the laser-target interactions, i.e., during the decay process of the electron sheath. According to the one-dimensional plasma expansion model proposed by Mora [56], the sheath charge $Q_{s}$ evolves over time $t$ approximately as $\left(1+\tau^{2}\right)^{-1 / 2}$, where $\tau=t / \tau_{s}, \tau_{s}$ is the characteristic decay time of sheath fields (which is proportional to $\left.1 / \omega_{p i}\right], \omega_{p i}=\left(n_{s} e^{2} / \varepsilon_{0} m_{i}\right)^{1 / 2}$ is the ion plasma frequency, and $n_{s}$ is the sheath electron density at the end of the laser interactions. For sufficiently longduration laser pulses, ions have gained adequate acceleration, and subsequently, the sheath front moves at an almost constant velocity, which approximately equals the speed of the maximum-energy ions, $v_{s}$. The equivalent transient current can be expressed as $\boldsymbol{J}(x, t)=Q_{s} v_{s} \delta\left(x-x_{s}\right) \mathbf{e}_{x}$, where $x_{s}$ is the position of the sheath front and $\mathbf{e}_{x}$ is the unit vector along the target normal. This decaying current induces SR. Under the far-field approximation [74], the SR spectral intensity is given by

$$
I_{\mathrm{SR}}=\frac{Q_{s 0}^{2} \beta_{s}^{2} \sin ^{2} \theta}{16 \pi^{3} \varepsilon_{0} c}\left|G_{\mathrm{SR}}\left(\omega \tau_{s}\right)\right|^{2}
$$

where $Q_{s 0}$ is the sheath charge at the moment the sheath field starts to decay, $\beta_{s}=v_{s} / c$, and $G_{\mathrm{SR}}(\omega)=$ $\omega \int 1 / \sqrt{1+t^{2}} e^{i \omega t} d t$.

Since the above three radiation processes do not entirely overlap in time and have much different spectra, the resultant total $\mathrm{THz}$ radiation can be approximately treated as the sum of their spectral intensities, $I_{\mathrm{THz}}=I_{\mathrm{TR}}+I_{\mathrm{BR}}+I_{\mathrm{SR}}$. Thereafter, one can obtain the $\mathrm{THz}$ spectra at given parameters or retrieve specific characteristic quantities from a known $\mathrm{THz}$ spectrum. Figures 1(c) and 1(d) are obtained with the following parameters: For the TR, an electron bunch is considered with a Boltzmann energy distribution at a temperature of $T_{e}=1 \mathrm{MeV}$, a divergence angle of $30^{\circ}$, and a Gaussian temporal distribution at a bunch duration $\tau_{b}$ varying from $0.1 \mathrm{ps}$ to $10 \mathrm{ps}$. Note that $\rho=5 \mathrm{~mm}$, and $Q_{\mathrm{esc}}=I_{e} \cdot \tau_{b}$, where $I_{e}=100 \mathrm{kA}$. For the BR, trapped electrons of energies less than $T_{e}$ are considered with the same temporal and energy distributions to the escaping electron bunch. Here, $Q_{\text {trap }}=3 Q_{\mathrm{esc}}, E_{\mathrm{sh}}=0.3 \mathrm{TV} / \mathrm{m}$, and, accordingly, $\tau_{r}=0.016 \mathrm{ps}$ for the $1-\mathrm{MeV}$ electrons. For the SR, $v_{s}=0.1 c$ (corresponding to a proton energy of $5 \mathrm{MeV}), \tau_{s}=0.1 \mathrm{ps}$, and $Q_{s 0}=Q_{\text {trap }}$. Note that $\theta=75^{\circ}$ for all radiation.

In the fitting of the experimental $\mathrm{THz}$ spectra, the electron bunch duration is taken to be the same as the laser pulse, and the experimentally measured electron temperature, angular distribution, and proton energy are used as input. Thus, $Q_{\mathrm{esc}}, Q_{s 0}$, and $n_{s}$ can be inferred from the fitting.

\section{B. Experimental configuration and data acquisition}

The experiment was carried out by employing the Vulcan laser system at the Rutherford Appleton Laboratory. The laser delivered a $p$-polarized picosecond pulse centered at a 1053-nm wavelength and with an adjustable pulse duration. After being focused by an $f / 3$ off-axis parabolic mirror onto the target at an incidence angle of $30^{\circ}$, about $20 \%$ laser energy was contained in a spot of $4 \mu \mathrm{m}$ in diameter, resulting in a peak laser intensity of about $7 \times$ $10^{19} \mathrm{~W} / \mathrm{cm}^{2}$ for the laser energy of about $70 \mathrm{~J}$ on target and a full-width-at-half-maximum (FWHM) pulse duration of about 1.5 ps. The targets used were mainly $100-\mu$ m-thick copper foils with a lateral size in the range of $0.3-3 \mathrm{~mm}$, mounted on an insulating stalk to mitigate the electromagnetic pulse (EMP) generation [55]. For the laser and target parameters used, ions (primarily protons) were accelerated via the target-normal sheath acceleration mechanism.

The electrons, protons, and $\mathrm{THz}$ radiation emitted from the target rear were independently characterized simultaneously [31]. A wraparound array of optical fibers, with an angular coverage of about $90^{\circ}$ at $5^{\circ}$ intervals, was used to sample electrons at different directions by detecting the electron-induced Cherenkov light in fibers [75]. An electron bunch escaping primarily in the target-normal direction with a FWHM divergence angle of about $30^{\circ}$ was observed. After calibration with wraparound image plate stacks, the electron charge was obtained by integrating the signal from each fiber loop, with consideration of the correction factor caused by the bend loss in the fiber. An electron spectrometer placed in the laser forward direction was used to measure the energy spectrum of the escaping electrons, typically showing a Boltzmann distribution with a temperature that was positively correlated to the laser intensity. Protons emitted in the rear target normal were detected with a Thomson parabola spectrometer. The proton charge was obtained by integrating the measured proton energy spectrum, and the total proton beam energy was evaluated by considering the proton beam's spatial distribution measured with stacked dosimetry films.

To sample the $\mathrm{THz}$ angular distribution, the radiation within a small solid angle at $75^{\circ}, 45^{\circ}$, and $-40^{\circ}$ with respect 
to the rear target normal was measured. Tests with holearrayed microwave filters, HRFZ silicon, aluminum foil, and dry paper were taken to confirm that the detected signal was from the THz radiation, rather than the EMP, scattered laser light, or x rays. See Supplemental Material [48] for the data of the $\mathrm{THz}$ angular distribution. Since the electrooptical sampling technique was inapplicable without an available femtosecond laser probe, a set of calibrated bandpass filters were used to characterize the discretized spectrum of the $\mathrm{THz}$ radiation at $75^{\circ}$. This filters-based spectrally-resolved detection scheme was frequently employed in high-power laser experiments, and its credibility was testified repeatedly with electro-optical measurements [33,37]. The $\mathrm{THz}$ radiation was split into multiple beams by high-resistivity silicon beam splitters. In each beam, narrowband $\mathrm{THz}$ bandpass filters with different central frequencies $\omega_{i}$ were inserted. The filtered $\mathrm{THz}$ beams were detected with cross-calibrated $\mathrm{THz}$ detectors (response range 0.1-250 THz). The whole $\mathrm{THz}$ detection system was housed in a grounded metal shielding box to avoid the unwanted interference by the EMP. The $\mathrm{THz}$ energy emitted from the target, $S_{0}$, within the transmission bandwidth of the bandpass filters, $\Delta \omega_{i}$, was retrieved from the signal recorded by the detector, $S_{\text {det }}$, via the following formula:

$S_{\text {det }}\left(\omega_{i}\right)=S_{0}\left(\omega_{i}\right) \cdot T_{\text {col }}\left(\omega_{i}\right) \cdot T_{\text {bs }}\left(\omega_{i}\right) \cdot T_{\text {filter }}\left(\omega_{i}\right) \cdot R_{\text {det }}\left(\omega_{i}\right)$

where $T_{\text {col }}, T_{\mathrm{bs}}$, and $T_{\text {filter }}$ represent the transmittance (at the frequency $\omega_{i}$ ) of the $\mathrm{THz}$ collection optics, silicon beam splitters, and the $\mathrm{THz}$ filter, respectively, and $R_{\text {det }}$ is the calibrated detector responsivity. The THz spectral intensity at $\omega_{i}$ was evaluated eventually as $I\left(\omega_{i}\right)=S_{0}\left(\omega_{i}\right) /\left(\Delta \omega_{i} \cdot \Delta \Omega\right)$. The THz radiation measured experimentally is very strong and has a tunable spectral profile, implying that it is neither incoherent radiation nor thermal emission.

\section{Evaluation for $\mathbf{T H z}$ parameters}

Given the good reproduction of the experimental measurements with the theoretical calculations (see Fig. 2 and Fig. S1 of Supplemental Material [48]), one can evaluate the total energy and peak power of $\mathrm{THz}$ radiation emitted from the target rear side by extrapolating the measured $\mathrm{THz}$ spectral and angular distributions with theoretical models. See Supplemental Material [48] for details on the evaluation of $\mathrm{THz}$ parameters. In view of the rather large divergence angle of $\mathrm{THz}$ radiation, the beam quality factor, $\mathrm{M}^{2}$, for the solid-plasma-based sources is taken conservatively to be 3 in the evaluation of brightness temperature. For the FEL and organic crystal-based sources, $\mathrm{M}^{2} \sim 1$, while for other sources, the $\mathrm{M}^{2}$ values are estimated by the ratio of the reported $\mathrm{THz}$ focal spot size to the central wavelength.

\section{Calculation of sheath electron density}

Based on the balance of energy fluxes, the density of energetic electrons produced at the target front, $n_{\text {front }}$, can be deduced as $\eta I_{L}=n_{\text {front }} v_{e} T_{e}$, where $\eta$ is the laser absorption efficiency, $I_{L}$ is the laser intensity, and $T_{e}$ and $v_{e}$ are the temperature and average velocity of electrons, respectively. After the ballistic propagation with a divergence angle of $\theta$ through the target thickness $d$, the electron density at the target rear is reduced as $n_{\text {rear }}=\left[r_{0} / r_{\text {rear }}\right]^{2} \cdot n_{\text {front }}$, where $r_{\text {rear }}=r_{0}+d \cdot \tan \theta$, and $r_{0}$ is the radius of the laser focal spot. The electrons spread out over the target surface almost at the speed of light, $c$. If the target size $\rho$ is smaller than the lateral extension scale of electrons, the electrons will bounce back from the target edges during the laser pulse, thus increasing the electron density. Considering this transverse refluxing effect, the transient sheath electron density at the end of laser pulse is evaluated as $n_{s}=\left[\max \left(r_{\text {rear }}+c \cdot \tau_{L}, \rho / 2\right) /(\rho / 2)\right]^{2} \cdot n_{\text {rear }}$.

Data associated with research published in this paper can be accessed at [76].

\section{ACKNOWLEDGMENTS}

We would like to thank the Vulcan laser team and the staff at the Central Laser Facility for laser operation and technical support. This work is supported by the National Natural Science Foundation of China (Grants No. 11520101003, No. 11721091, and No. 11827807), the Science Challenge Project (No. TZ2016005), the Strategic Priority Research Program of the Chinese Academy of Sciences (Grants No. XDA25010000 and No. XDB16010200), the National Key Research and Development Program of China (Grant No. 2018YFA0404801), and the Newton UK grant. P. M. and D. N. acknowledge support from EPSRC (Grants No. EP/R006202/1 and No. EP/K022415/1).

[1] X. C. Zhang, A. Shkurinov, and Y. Zhang, Extreme Terahertz Science, Nat. Photonics 11, 16 (2017).

[2] S. S. Dhillon et al., The 2017 Terahertz Science and Technology Roadmap, J. Phys. D 50, 043001 (2017).

[3] H. A. Hafez, X. Chai, A. Ibrahim, S. Mondal, D. Férachou, X. Ropagnol, and T. Ozaki, Intense Terahertz Radiation and Their Applications, J. Opt. 18, 093004 (2016).

[4] T. Kampfrath, K. Tanaka, and K. A. Nelson, Resonant and Nonresonant Control over Matter and Light by Intense Terahertz Transients, Nat. Photonics 7, 680 (2013).

[5] J. L. LaRue, T. Katayama, A. Lindenberg, A. S. Fisher, H. Öström, A. Nilsson, and H. Ogasawara, THz-Pulse-Induced Selective Catalytic CO Oxidation on Ru, Phys. Rev. Lett. 115, 036103 (2015). 
[6] E. A. Nanni, W. R. Huang, K.-H. Hong, K. Ravi, A. Fallahi, G. Moriena, R. J. Dwayne Miller, and F. X. Kärtner, Terahertz-Driven Linear Electron Acceleration, Nat. Commun. 6, 8486 (2015).

[7] D. Zhang et al., Segmented Terahertz Electron Accelerator and Manipulator (STEAM), Nat. Photonics 12, 336 (2018).

[8] E. Curry, S. Fabbri, J. Maxson, P. Musumeci, and A. Gover, Meter-Scale Terahertz-Driven Acceleration of a Relativistic Beam, Phys. Rev. Lett. 120, 094801 (2018).

[9] C. Kealhofer, W. Schneider, D. Ehberger, A. Ryabov, F. Krausz, and P. Baum, All-Optical Control and Metrology of Electron Pulses, Science 352, 429 (2016).

[10] L. Zhao et al., Terahertz Streaking of Few-Femtosecond Relativistic Electron Beams, Phys. Rev. X 8, 021061 (2018).

[11] P. Weightman, Prospects for the Study of Biological Systems with High Power Sources of Terahertz Radiation, Phys. Biol. 9, 053001 (2012).

[12] N. Stojanovic and M. Drescher, Accelerator- and LaserBased Sources of High-Field Terahertz Pulses, J. Phys. B 46, 192001 (2013).

[13] S. Bielawski et al., Tunable Narrowband Terahertz Emission from Mastered Laser-Electron Beam Interaction, Nat. Phys. 4, 390 (2008).

[14] Y. Shen, X. Yang, G. L. Carr, Y. Hidaka, J. B. Murphy, and $\mathrm{X}$. Wang, Tunable Few-Cycle and Multicycle Coherent Terahertz Radiation from Relativistic Electrons, Phys. Rev. Lett. 107, 204801 (2011).

[15] G. N. Kulipanov et al., Research Highlights from the Novosibirsk $400 \mathrm{~W}$ Average Power THz FEL, Terahertz Sci. Technol. 1, 107 (2008).

[16] Z. Wu, A. S. Fisher, J. Goodfellow, M. Fuchs, D. Daranciang, M. Hogan, H. Loos, and A. Lindenberg, Intense Terahertz Pulses from SLAC Electron Beams Using Coherent Transition Radiation, Rev. Sci. Instrum. 84, 022701 (2013).

[17] C. Evain et al., Stable Coherent Terahertz Synchrotron Radiation from Controlled Relativistic Electron Bunches, Nat. Phys. 15, 635 (2019).

[18] C. Vicario, A. V. Ovchinnikov, S. I. Ashitkov, M. B. Agranat, V. E. Fortov, and C. P. Hauri, Generation of $0.9-\mathrm{mJ} \mathrm{THz}$ Pulses in DSTMS Pumped by a $\mathrm{Cr}: \mathrm{Mg}_{2} \mathrm{SiO}_{4}$ Laser, Opt. Lett. 39, 6632 (2014).

[19] M. Shalaby and C. P. Hauri, Demonstration of a LowFrequency Three-Dimensional Terahertz. Bullet with Extreme Brightness, Nat. Commun. 6, 5976 (2015).

[20] J. A. Fülöp, Z. Ollmann, Cs. Lombosi, C. Skrobo, S. Klingebie, L. Pálfalvi, F. Krausz, S. Karsch, and J. Hebling, Efficient Generation of THz pulses with $0.4 \mathrm{~mJ}$ Energy, Opt. Express 22, 20155 (2014).

[21] S.-W. Huang, E. Granados, W. R. Huang, K.-H. Hong, L. E. Zapata, and F. X. Kärtner, High Conversion Efficiency, High Energy Terahertz Pulses by Optical Rectification in Cryogenically Cooled Lithium Niobate, Opt. Lett. 38, 796 (2013).

[22] D. H. Auston, K. P. Cheung, and P. R. Smith, Picosecond Photoconducting Hertzian Dipoles, Appl. Phys. Lett. 45, 284 (1984).

[23] H. Hamster, A. Sullivan, S. Gordon, W. White, and R. W. Falcone, Subpicosecond Electromagnetic Pulses from Intense Laser-Plasma Interaction, Phys. Rev. Lett. 71, 2725 (1993).
[24] W. P. Leemans et al., Observation of Terahertz Emission from a Laser-Plasma Accelerated Electron Bunch Crossing a Plasma-Vacuum Boundary, Phys. Rev. Lett. 91, 074802 (2003).

[25] K. Y. Kim, A. J. Taylor, J. H. Glownia, and G. Rodriguez, Coherent Control of Terahertz Supercontinuum Generation in Ultrafast Laser-Gas Interactions, Nat. Photonics 2, 605 (2008).

[26] D. Kuk, Y. J. Yoo, E. W. Rosenthal, N. Jhajj, H. M. Milchberg, and K. Y. Kim, Generation of Scalable Terahertz Radiation from Cylindrically Focused Two-Color Laser Pulses in Air, Appl. Phys. Lett. 108, 121106 (2016).

[27] M. Clerici et al., Wavelength Scaling of Terahertz Generation by Gas Ionization, Phys. Rev. Lett. 110, 253901 (2013).

[28] I. Dey et al., Highly Efficient Broadband Terahertz. Generation from Ultrashort Laser Filamentation in Liquids, Nat. Commun. 8, 1184 (2017).

[29] Q. Jin, Y. E. K. Williams, J. Dai, and X. C. Zhang, Observation of Broadband Terahertz Wave Generation from Liquid Water, Appl. Phys. Lett. 111, 071103 (2017).

[30] G. Q. Liao and Y. T. Li, Review of Intense Terahertz. Radiation from Relativistic Laser-Produced Plasmas, IEEE Trans. Plasma Sci. 47, 3002 (2019).

[31] G. Q. Liao et al., Multimillijoule Coherent Terahertz Bursts from Picosecond Laser-Irradiated Metal Foils, Proc. Natl. Acad. Sci. U. S. A. 116, 3994 (2019).

[32] A. Gopal et al., Observation of Energetic Terahertz Pulses from Relativistic Solid Density Plasmas, New J. Phys. 14, 083012 (2012).

[33] A. Gopal et al., Observation of Gigawatt-Class THz Pulses from a Compact Laser-Driven Particle Accelerator, Phys. Rev. Lett. 111, 074802 (2013).

[34] G. Q. Liao et al., Demonstration of Coherent Terahertz Transition Radiation from Relativistic Raser-Solid Interactions, Phys. Rev. Lett. 116, 205003 (2016).

[35] Z. Jin et al., Highly Efficient Terahertz Radiation from a Thin Foil Irradiated by a High-Contrast Laser Pulse, Phys. Rev. E 94, 033206 (2016).

[36] A. S. Kuratov, A. V. Brantov, Yu. M. Aliev, and V. Yu. Bychenkov, Terahertz Radiation in Laser-Induced Charge Separation in the Irradiated Plasma Target, Quantum Electron. 46, 1023 (2016).

[37] S. Herzer et al., An Investigation on THz Yield from LaserProduced Solid Density Plasmas at Relativistic Laser Intensities, New J. Phys. 20, 063019 (2018).

[38] A. H. Woldegeorgis, B. Beleites, F. Ronneberger, R. Grosse, and A. Gopal, Investigating the Influence of Incident Laser Wavelength and Polarization on Particle Acceleration and Terahertz Generation, Phys. Rev. E 98, 061201(R) (2018).

[39] A. Gopal, A. Woldegeorgis, S. Herzer, and M. Almassarani, Spatiotemporal Visualization of the Terahertz Emission during High-power Laser-Matter Interaction, Phys. Rev. E 100, 053203 (2019).

[40] A. Woldegeorgis, S. Herzer, M. Almassarani, S. Marathapalli, and A. Gopal, Modeling Terahertz Emission from the Target Rear Side during Intense Laser-Solid Interactions, Phys. Rev. E 100, 053204 (2019).

[41] P. Gibbon, Short Pulse Laser Interactions with Matter: An Introduction (Imperial College Press, London, 2005). 
[42] J. Zheng, K. A. Tanaka, T. Miyakoshi, Y. Kitagawa, R. Kodama, T. Kurahashi, and T. Yamanaka, Theoretical Study of Transition Radiation from Hot Electrons Generated in the Laser-Solid Interaction, Phys. Plasmas 10, 2994 (2003).

[43] C. B. Schroeder, E. Esarey, J. van Tilborg, and W. P. Leemans, Theory of Coherent Transition Radiation Generated at a Plasma-Vacuum Interface, Phys. Rev. E 69, 016501 (2004).

[44] A. Macchi, M. Borghesi, and M. Passoni, Ion Acceleration by Superintense Laser-Plasma Interaction, Rev. Mod. Phys. 85, 751 (2013).

[45] S. Inoue, S. Tokita, K. Otani, M. Hashida, M. Hata, H. Sakagami, T. Taguchi, and S. Sakabe, Autocorrelation Measurement of Fast Electron Pulses Emitted through the Interaction of Femtosecond Laser Pulses with a Solid Target, Phys. Rev. Lett. 109, 185001 (2012).

[46] L. Romagnani et al., Dynamics of Electric Fields Driving the Laser Acceleration of Multi-MeV Protons, Phys. Rev. Lett. 95, 195001 (2005).

[47] S. Buffechoux et al., Hot Electrons Transverse Refluxing in Ultraintense Laser-Solid Interactions, Phys. Rev. Lett. 105, 015005 (2010).

[48] See Supplemental Material at http://link.aps.org/ supplemental/10.1103/PhysRevX.10.031062 for the data on the $\mathrm{THz}$ angular distribution and the evaluation of THz parameters.

[49] P. Shukla, J. Lawrence, and Y. Zhang, Understanding Laser Beam Brightness: A Review and New Prospective in Material Processing, Opt. Laser Technol. 75, 40 (2015).

[50] Y. Shibata, T. Takahashi, T. Kanai, K. Ishi, M. Ikezawa, J. Ohkuma, S. Okuda, and T. Okada, Diagnostics of an Electron Beam of a Linear Accelerator Using Coherent Transition Radiation, Phys. Rev. E 50, 1479 (1994).

[51] J. van Tilborg, C. B. Schroeder, C. V. Filip, Cs. Tóth, C. G. R. Geddes, G. Fubiani, R. Huber, R. A. Kaindl, E. Esarey, and W. P. Leemans, Temporal Characterization of Femtosecond Laser-Plasma-Accelerated Electron Bunches Using Terahertz Radiation, Phys. Rev. Lett. 96, 014801 (2006).

[52] A. D. Debus et al., Electron Bunch Length Measurements from Laser-Accelerated Electrons Using Single-Shot $\mathrm{THz}$ Time-Domain Interferometry, Phys. Rev. Lett. 104, 084802 (2010).

[53] J.-L. Dubois et al., Target Charging in Short-PulseLaser-Plasma Experiments, Phys. Rev. E 89, 013102 (2014).

[54] R. Pompili et al., Femtosecond Dynamics of Energetic Electrons in High Intensity Laser-Matter Interactions, Sci. Rep. 6, 35000 (2016).

[55] A. Poyé et al., Physics of Giant Electromagnetic Pulse Generation in Short-Pulse Laser Experiments, Phys. Rev. E 91, 043106 (2015).

[56] P. Mora, Plasma Expansion into a Vacuum, Phys. Rev. Lett. 90, 185002 (2003).

[57] P. Antici et al., Hot and Cold Electron Dynamics Following High-Intensity Laser Matter Interaction, Phys. Rev. Lett. 101, 105004 (2008).

[58] O. Jäckel, J. Polz, S. M. Pfotenhauer, H.-P. Schlenvoigt, H. Schwoerer, and M. C. Kaluza, All-Optical Measurement of the Hot Electron Sheath Driving Laser Ion Acceleration from Thin Foils, New J. Phys. 12, 103027 (2010).

[59] P. McKenna et al., Lateral Electron Transport in High-Intensity Laser-Irradiated Foils Diagnosed by Ion Emission, Phys. Rev. Lett. 98, 145001 (2007).

[60] O. Tresca et al., Controlling the Properties of Ultraintense Laser-Proton Sources Using Transverse Refluxing of Hot Electrons in Shaped Mass-Limited Targets, Plasma Phys. Controlled Fusion 53, 105008 (2011).

[61] A. J. Mackinnon, Y. Sentoku, P. K. Patel, D. W. Price, S. Hatchett, M. H. Key, C. Andersen, R. Snavely, and R. R. Freeman, Enhancement of Proton Acceleration by HotElectron Recirculation in Thin Foils Irradiated by Ultraintense Laser Pulses, Phys. Rev. Lett. 88, 215006 (2002).

[62] J. Fuchs et al., Laser-Driven Proton Scaling Laws and New Paths Towards Energy Increase, Nat. Phys. 2, 48 (2006).

[63] J. J. Santos et al., Fast Electron Transport in Ultraintense Laser Pulse Interaction with Solid Targets by Rear-Side Self-Radiation Diagnostics, Phys. Rev. Lett. 89, 025001 (2002).

[64] L. Robson et al., Scaling of Proton Acceleration Driven by Petawatt-Laser-Plasma Interactions, Nat. Phys. 3, 58 (2007).

[65] A. Yogo et al., Boosting Laser-Ion Acceleration with Multi-Picosecond Pulses, Sci. Rep. 7, 42451 (2017).

[66] D. Nicoletti and A. Cavalleri, Nonlinear Light-Matter Interaction at Terahertz Frequencies, Adv. Opt. Photonics 8, 401 (2016).

[67] L. J. Wong, A. Fallahi, and F. X. Kärtner, Compact Electron Acceleration and Bunch Compression in $\mathrm{THz}$. Waveguides, Opt. Express 21, 9792 (2013).

[68] P. Hamm, M. Meuwly, S. L. Johnson, P. Beaud, and U. Staub, Perspective: THz-Driven Nuclear Dynamics from Solids to Molecules, Struct. Dyn. 4, 061601 (2017).

[69] G. A. Mourou, T. Tajima, and S. V. Bulanov, Optics in the Relativistic Regime, Rev. Mod. Phys. 78, 309 (2006).

[70] B. Wolter, M. G. Pullen, M. Baudisch, M. Sclafani, M. Hemmer, A. Senftleben, C. D. Schröter, J. Ullrich, R. Moshammer, and J. Biegert, Strong-Field Physics with Mid-IR Fields, Phys. Rev. X 5, 021034 (2015).

[71] J. Weisshaupt, V. Juvé, M. Holtz, S. A. Ku, M. Woerner, T. Elsaesser, S. Ališauskas, A. Pugžlys, and A. Baltuška, HighBrightness Table-Top Hard X-Ray Source Driven by Sub100-Femtosecond Mid-Infrared Pulses, Nat. Photonics 8, 927 (2014).

[72] Z. Samsonova et al., Relativistic Interaction of Long-Wavelength Ultrashort Laser Pulses with Nanowires, Phys. Rev. X 9, 021029 (2019).

[73] S. M. Teo, B. K. Ofori-Okai, C. A. Werley, and K. A. Nelson, Single-Shot $\mathrm{THz}$ Detection Techniques Optimized for Multidimensional THz Spectroscopy, Rev. Sci. Instrum. 86, 051301 (2015).

[74] J. D. Jackson, Classical Electrodynamics, 3rd ed. (Wiley, New York, 1999).

[75] H. Liu et al., Cherenkov Radiation-Based Optical Fibre Diagnostics of Fast Electrons Generated in Intense LaserPlasma Interactions, Rev. Sci. Instrum. 89, 083302 (2018).

[76] Data associated with research published in this paper can be accessed at https://doi.org/10.5286/edata/741. 